\title{
Nonlinear Deformation of Flexible Orthotropic Shells of Variable Thickness in an Unsteady Magnetic Field
}

\author{
Zafar Abdullaev*, Sayibdjan Mirzaev and Sobir Mavlanov \\ Department of Information Technologies, Tashkent institute of irrigation and agricultural \\ mechanization engineers, Tashkent, 100000, Uzbekistan
}

\begin{abstract}
The analysis of the stress state of a flexible orthotropic shell under the influence of a time-varying mechanical force and a timevarying external electric current is performed, taking into account the mechanical and electromagnetic orthotropy. The effect of thickness on the stress-strain state of the orthotropic shell is investigated. The results obtained indicate the influence of thickness on the deformation of the shell and the need to take this factor into account in the design schemes.
\end{abstract}

\section{Introduction}

The development of research in the theory of magnetoelasticity is associated with the solution of many important problems of modern technology. Such tasks arise in the development of electromagnetic pumps, magnetohydrodynamic accelerators, instrumentation that works with electromagnetic fields, the imposition of magnetic fields in controlling the movement of plasma, in the flow in an elastic shell, the calculation of protective shields, atomic reactors, setting up some physical experiments, etc.

The construction of optimal designs of modern technology operating in magnetic fields is associated with the wide use of structural elements, such as flexible thin-walled shells. The effect of non-stationary fields on metallic thin-walled elements leads to the appearance of bulk electromagnetic forces, capable of causing large deformations of structures under certain field parameters. Recently, the question of determining the stress state of flexible orthotropic shells operating in an alternating magnetic field with regard to orthotropic electrical conductivity has raised considerable interest.

\section{Nonlinear formulation of the problem. Basic equations}

Consider the nonlinear behavior of an orthotropic current-carrying conical shell of beryllium of variable thickness, changing in the meridional direction according to the law $h=5 \cdot 10^{-4}\left(1-\alpha^{S} / S_{N}\right) M$. We believe that the shell is under the influence of mechanical force $P_{\zeta}=5 \cdot 10^{3} \sin \omega t^{H} / M^{2}$, third-party electric current $J_{\theta C T}=-5$. $10^{5} \sin \omega t{ }^{A} / M^{2}$, and external magnetic field $B_{S 0}=0.1$ л, and that the shell has a finite

\footnotetext{
*Corresponding author: sobitoy@mai.ru, smirzaev@yandex.ru
} 
orthotropic conductivity $\sigma\left(\sigma_{1}, \sigma_{2}, \sigma_{3}\right)$.

We assume that the external electric current in the unperturbed state is uniformly distributed over the shell, i.e. External current density does not depend on the coordinates. In this case, the shell is affected by a combined load consisting of the ponderomotive Lorentz force and the mechanical force. Small radius contour $S=S_{0}$ hinged, and the second circuit $S=S_{N}$ - free in the meridional direction.

Note that in the case under consideration an arbitrary second-order surface has three mutually perpendicular axes of the second order and these axes can be arranged parallel to the second-order crystallographic axes, and the second-order characteristic surface has all the symmetry elements that can be found in the classes of the orthorhombic system $[2,3]$.

Suppose that the geometric and mechanical characteristics of the body are such that to describe the process of deformation, we apply the variant of the geometrically nonlinear theory of thin shells in the quadratic approximation. Also assume that with respect to the electric field strength $\vec{E}$ and magnetic field strength $\vec{H}$ electromagnetic hypotheses are performed $[1,4]$ :

$$
\begin{gathered}
E_{1}=E_{1}(\alpha, \beta, t) ; \quad E_{2}=E_{2}(\alpha, \beta, t) ; E_{3}=\frac{\partial u_{2}}{\partial t} B_{1}-\frac{\partial u_{1}}{\partial t} B_{2} \\
J_{1}=J_{1}(\alpha, \beta, t) ; \quad J_{2}=J_{2}(\alpha, \beta, t) ; J_{3}=0 \\
H_{1}=\frac{1}{2}\left(H_{1}^{+}+H_{1}^{-}\right)+\frac{z}{h}\left(H_{1}^{+}-H_{1}^{-}\right) \\
H_{2}=\frac{1}{2}\left(H_{2}^{+}+H_{2}^{-}\right)+\frac{z}{h}\left(H_{2}^{+}-H_{2}^{-}\right) ; H_{3}=H_{3}(\alpha, \beta, t) .
\end{gathered}
$$

$u_{i}$ - shell vector displacement components; $E_{i}, H_{i}-$ components of the vectors of the electric and magnetic fields of the shell; $J_{i}$ - eddy current components; $H_{i}^{ \pm}-$ tangential components of the magnetic field on the surfaces of the shell; $h-$ shell thickness.

These assumptions are some electrodynamic analog of the hypothesis of undeformable normals and together with the latter constitute hypotheses of magnetoelasticity of thin bodies. The adoption of these hypotheses allows us to reduce the problem of the deformation of a three-dimensional body to the problem of the deformation of an arbitrarily selected coordinate surface.

The developed methodology for the numerical solution of a new class of related problems of magnetoelasticity of the theory of orthotropic conical shells of revolution with orthotropic electrical conductivity is based on the sequential application of Newmark's finite-difference scheme, the linearization method and discrete orthogonalization [4-6].

To effectively use the proposed technique, we assume that when an external magnetic field appears, there are no sharp skin effects along the shell thickness and an electromagnetic process along the coordinate $\zeta$ quickly goes to a mode close to steady. This leads to restrictions on the nature of changes in the external magnetic field and on the geometric and electrophysical parameters of the shell.

$$
\frac{\tau}{h^{2} \sigma \mu}>1
$$

$\tau-$ characteristic time of the magnetic field. In case of non-fulfillment of this 
condition, only the equations of shell movement under the action of magnetic pressure should be considered.

In this formulation, the system of equations describing the nonlinear oscillations of a flexible current-carrying orthotropic conical shell of variable thickness on the corresponding time layer, according to [5, 6], after applying the quasi-linearization method, takes the form

$$
\begin{aligned}
& \frac{d u^{(k+1)}}{d m}=\frac{1-v_{S} v_{\theta}}{\rho e_{S} h} N_{S}^{(k+1)}-\frac{v_{\theta} \cos \varphi}{\rho r} u^{(k+1)}-\frac{v_{\theta} \sin \varphi}{\rho r} w^{(k+1)}+\frac{1}{2 \rho}\left(\theta_{S}^{(k)}\right)^{2}-\theta_{S}^{(k+1)} \theta_{S}^{(k)} \\
& \frac{d w^{(k+1)}}{d m}=-\frac{\theta_{S}^{(k+1)}}{\rho} ; \frac{d \theta_{S}^{(k+1)}}{d m}=\frac{12\left(1-v_{S} v_{\theta}\right)}{\rho e_{S} h^{3}} M_{S}^{(k+1)}-\frac{v_{\theta} \cos \varphi}{\rho r} \theta_{S}^{k+1} \\
& \frac{d N_{S}^{(k+1)}}{d m}=\frac{\cos \varphi}{\rho r}\left[\left(v_{\theta} \frac{e_{\theta}}{e_{S}}-1\right) N_{S}^{(k+1)}+e_{\theta} h\left(\frac{\cos \varphi}{r} u^{(k+1)}+\frac{\sin \varphi}{r} w^{(k+1)}\right)\right] \\
& -\frac{P_{S}^{(k+1)}}{\rho}+\frac{h}{\rho} J_{\theta C T} B_{\zeta}^{(k+1)}-\frac{\sigma_{1} h}{\rho}\left[\left(-E_{\theta}^{(k)} B_{\zeta}^{(k)}+E_{\theta}^{(k+1)} B_{\zeta}^{(k)}+E_{\theta}^{(k)} B_{\zeta}^{(k+1)}\right)+\right. \\
& 0.5\left\{-\left(\dot{w}^{(t+\Delta t)}\right)^{(k)} B_{\zeta}^{(k)}+\left(\dot{w}^{(t+\Delta t)}\right)^{(k+1)} B_{\zeta}^{(k)}+\left(\dot{w}^{(t+\Delta t)}\right)^{(k)} B_{\zeta}^{(k+1)}\right\}\left(B_{S}^{+}+B_{S}^{-}\right)- \\
& \left.-\left\{-\left(B_{\zeta}^{(k)}\right)^{2}\left(\dot{u}^{(t+\Delta t)}\right)^{k}+\left(B_{\zeta}^{(k)}\right)^{2}\left(\dot{u}^{(t+\Delta t)}\right)^{(k+1)}+2 B_{\zeta}^{(k+1)} B_{\zeta}^{(k)}\left(\dot{u}^{(t+\Delta t)}\right)^{k}\right\}\right]+ \\
& h\left(\ddot{u}^{(t+\Delta t)}\right)^{(k+1)} \\
& \frac{d \mathrm{Q}_{S}^{(k+1)}}{d m}=-\frac{\cos \varphi}{\rho r} \mathrm{Q}_{S}^{(k+1)}+v_{s} \frac{e_{\theta}}{e_{S}} \frac{\sin \varphi}{\rho r} N_{S}^{(k+1)}+\frac{e_{\theta} h}{\rho} \frac{\sin \varphi}{r}\left(\frac{\cos \varphi}{r} u^{(k+1)}+\frac{\sin \varphi}{r} w^{(k+1)}\right)- \\
& -\frac{P_{\zeta}^{(k+1)}}{\rho}-0.5 \frac{h}{\rho} J_{\theta C T}\left(B_{S}^{+}+B_{S}^{-}\right)-\frac{\sigma_{3} h}{\rho}\left[-0.5 E_{\theta}^{(k+1)}\left(B_{S}^{+}+B_{S}^{-}\right)-0.25\left(\dot{w}^{(t+\Delta t)}\right)^{(k+1)}\left(B_{S}^{+}+\right.\right. \\
& \left.B_{S}^{-}\right)^{2} \quad(3) \\
& -\frac{1}{12}\left(\dot{w}^{(t+\Delta t)}\right)^{(k+1)}\left(B_{S}^{+}-B_{S}^{-}\right)^{2}+0.5\left\{-\left(\dot{u}^{(t+\Delta t)}\right)^{k} B_{\zeta}^{(k)}+\left(\dot{u}^{(t+\Delta t)}\right)^{(k+1)} B_{\zeta}^{(k)}+\right. \\
& \left.\left(\dot{u}^{(t+\Delta t)}\right)^{(k)} B_{\zeta}^{(k+1)}\right\} \\
& \left(B_{S}^{+}+B_{S}^{-}\right)+\frac{h}{12}\left\{-\left(\dot{\theta}^{(t+\Delta t)}\right)^{(k)} B_{\zeta}^{(k)}-\left(\dot{\theta}^{(t+\Delta t)}\right)^{(k+1)} B_{\zeta}^{(k)}+\left(\dot{\theta}^{(t+\Delta t)}\right)^{(k)} B_{\zeta}^{(k+1)}\right\}\left(B_{S}^{+}\right. \\
& \left.\left.+B_{S}^{-}\right)\right]+ \\
& h\left(\ddot{W}^{(t+\Delta t)}\right)^{(k+1)} ; \\
& \frac{d M_{S}^{(k+1)}}{d m}=\frac{\cos \varphi}{\rho r}\left[\left(v_{s} \frac{e_{\theta}}{e_{S}}-1\right) M_{S}^{(k+1)}+\frac{e_{\theta} h^{3}}{12} \frac{\cos \varphi}{r} \theta_{S}^{(k+1)}\right]+\frac{\mathrm{Q}_{S}^{(k+1)}}{\rho}
\end{aligned}
$$




$$
\begin{aligned}
& +\frac{1}{\rho}\left(-N_{S}^{(k)} \theta_{S}^{(k)}+N_{S}^{(k+1)} \theta_{S}^{k}+N_{S}^{(k)} \theta_{S}^{k+1}\right)-v_{S} \frac{e_{\theta}}{e_{S}} \frac{\sin \varphi}{\rho r}\left(-M_{S}^{k} \theta_{S}^{K}+M_{S}^{k+1} \theta_{S}^{k}+M_{S}^{k} \theta_{S}^{k+1}\right)- \\
& -\frac{e_{\theta} h^{3}}{12} \frac{\sin \varphi \cos \varphi}{\rho r^{2}}\left[-\left(\theta_{S}^{(k)}\right)^{2}+2 \theta_{S}^{(k+1)} \theta_{S}^{k}\right]+\frac{h^{3}}{12 \rho}\left(\ddot{\theta}^{(t+\Delta t)}\right)^{(k+1)} ; \\
& \frac{d B_{\zeta}^{(k+1)}}{d m}=-\frac{\sigma_{2} \mu}{\rho}\left[E_{\theta}^{(k+1)}+0.5\left(\dot{w}^{(t+\Delta t)}\right)^{(k+1)}\left(B_{S}^{+}+B_{S}^{-}\right.\right. \\
& \left.\left\{-\left(\dot{u}^{(t+\Delta t)}\right)^{(k)} B_{\zeta}^{(k)}+\left(\dot{u}^{(t+\Delta t)}\right)^{(k+1)} B_{\zeta}^{(k)}+\left(\dot{u}^{(t+\Delta t)}\right)^{k} B_{\zeta}^{(k+1)}\right\}\right]+\frac{B_{S}^{+}-B_{S}^{-}}{\rho h} ; \\
& \frac{d E_{\theta}^{(k+1)}}{d m}=-\frac{1}{\rho}\left(\dot{B}_{\zeta}^{(t+\Delta t)}\right)^{(k+1)}-\frac{\cos \varphi}{\rho r} E_{\theta}^{(k+1)},(k=0,1,2, \ldots \ldots .) .
\end{aligned}
$$

In this case, the boundary conditions we write in the form

$$
\begin{aligned}
& u=0, \quad w=0, \quad M_{S}=0, \quad B_{\zeta}=0.3 \sin \omega t, \quad S=S_{0}=0, \\
& w=0, \quad \theta_{S}=0, \quad N_{S}=0, \quad B_{\zeta}=0, \quad S=S_{N}=0.5 M .
\end{aligned}
$$

Initial conditions take the form

$$
\left.\vec{N}(s, t)\right|_{t=0}=0,\left.\quad u(s, t)\right|_{t=0}=0, \quad w(s, t) \dot{\mid}_{t=0}=0
$$

$N_{S}, N_{\theta}-$ meridional and district forces; $S$ - shear force; $Q_{s}-$ shear force; $M_{S}, M_{\theta}-$ bending moments; $u, w-$ displacement and deflection; $\theta_{S}-$ normal angle; $P_{S}, P_{\zeta}-$ mechanical load components; $E_{\theta}$ - circumferential component of the electric field; $B_{\zeta}-$ normal magnetic induction; $B_{s}^{+}, B_{s}^{-}-$known magnetic induction components on shell surfaces; $J_{\theta \mathrm{cm}}$ - component of the density of electric current from an external source; $e_{s}, e_{\theta}-$ elastic moduli for directions $s, \theta-$ respectively; $v_{s}, v_{\theta}-$ Puasson's ratios characterizing lateral compression under tension in the direction of the axes of coordinates; $\mu$ - magnetic permeability; $\omega-$ circular frequency; $\sigma_{1}, \sigma_{2}, \sigma_{3}-$ main components of conductivity tensor.

\section{Numeric example. Analysis of the results}

We study the behavior of an orthotropic shell depending on the change in the thickness of the shell. Problem for an orthotropic beryllium cone of variable thickness $h=5$. $10^{-4}\left(1-\alpha^{S} / s_{N}\right) M \quad$ calculated for different parameter values $\alpha=$ $\{0.2 ; 0.3 ; 0.4 ; 0.5\}$ characterizing the thickness variability in the meridional direction.

When solving a problem, the parameters take the following values:

$$
\begin{gathered}
S_{0}=0, S_{N}=0,5 M, h=5 \cdot 10^{-4}\left(1-\alpha^{S} / S_{N}\right) M, r=r_{0}+\cos \varphi ; r_{0}=0.5 M, \omega \\
=314.16 c^{-1},
\end{gathered}
$$




$$
\begin{gathered}
\rho=2300 \mathrm{k} \Gamma /_{M^{3}}, B_{S}^{+}=B_{S}^{-}=0.5 \text { Тл. } \varphi=30^{\circ}, B_{\text {so }}=0.1 \text { лл, } \\
\mu=1.256 \cdot 10^{-6 \Gamma \mathrm{H}} / M_{M}, J_{\theta C T}=-5 \cdot 10^{5} \sin \omega t A / M^{2}, \sigma_{1}=0.279 \cdot 10^{8}(O \mathrm{M} \cdot \mathrm{M})^{-1} \\
\sigma_{2}=0.321 \cdot 10^{8}(\mathrm{OM} \cdot \mathrm{M})^{-1}, \sigma_{3}=1.36 \cdot 10^{8}(\mathrm{OM} \cdot \mathrm{M})^{-1}, \quad v_{S}=0.33, \quad v_{\theta}=0.09, \\
P_{\zeta}=5 \cdot 10^{3} \sin \omega t \mathrm{H} / \mathrm{M}^{2}, e_{S}=28.8 \cdot 10^{10} \mathrm{H} / \mathrm{M}^{2}, e_{\theta}=33.53 \cdot 10^{10} \mathrm{H} / \mathrm{M}^{2}
\end{gathered}
$$

The solution of the problem was on the time interval $\tau=0 \div 10^{-2} c$, time integration step is equal to $\Delta t=1 \cdot 10^{-3} c$.

In the case considered, the anisotropy of the electrical resistivity of beryllium is $\eta_{3} / \eta_{1}=4.07$.

In the figures below, the graphs $(1,2,3,4)$ correspond to the values of the parameter $\alpha=\{0.2 ; 0.3 ; 0.4 ; 0.5\}$.

In fig.1. deflection distribution shown $w$ along the shell meridian at time $t=5 \cdot 10^{-3} c$. for different parameter values $\alpha$.

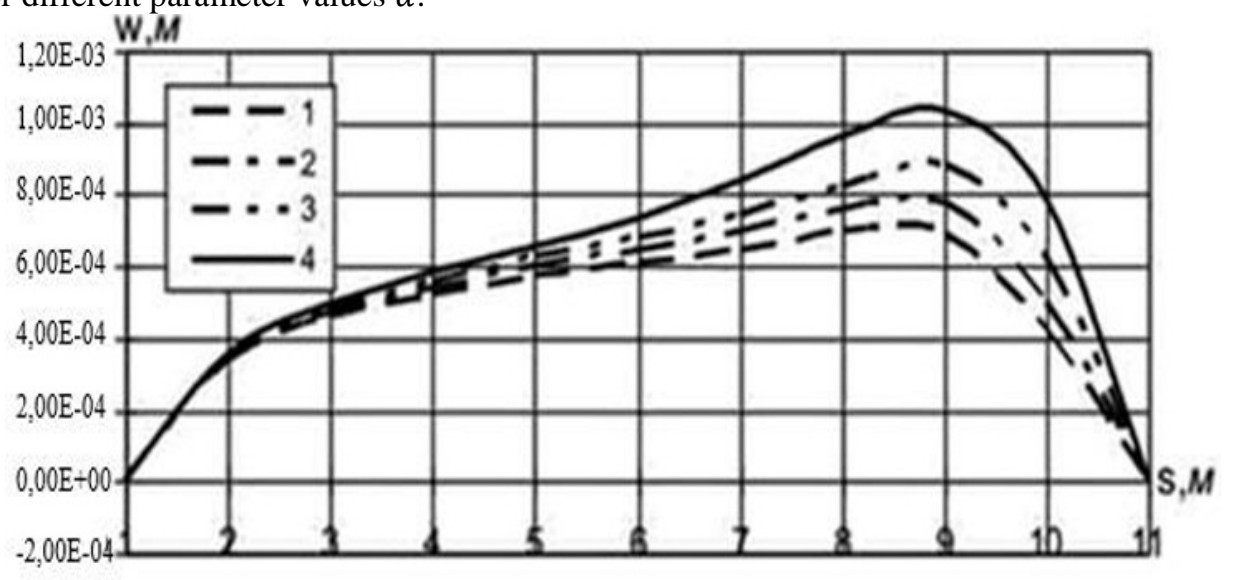

1. $\alpha=0.2 ; 2-\alpha=0.3 ; 3-\alpha=0.4 ; 4-\alpha=0.5$.

Fig. 1. Distribution by $s$ at the moment of time $w$ by $s$ at the moment of time $t=5 \cdot 10^{-3} c$ at different values of the parameter $\alpha$.

It has been established that the maximum values of the deflections along the shell arise approximately in the vicinity of $s=0.4 \mathrm{M}$.

This is explained by the fact that, according to the boundary conditions, the left end is pivotally fixed, and the right end of the shell is free in the meridional direction.

In addition, the thickness of the shell from the left end to the right end decreases to 2 times when $\alpha=0.5$. Therefore, the maximum deflection values occur near the right end of the shell.

When taking into account the effect of thickness, the stress of the conical shell was considered as the sum of the mechanical stresses and maxwell stresses, i.e. the general stress state was taken into account.

In fig. 2 and 3 show the distribution of maximum stress values $\left(\sigma_{22}^{+}+T_{22}^{+}\right)$and $\left(\sigma_{22}^{-}+T_{22}^{-}\right)$along the shell meridian at time $t=5 \cdot 10^{-3} \mathrm{c}$ over the outer and inner surfaces of the shell for different parameter values $\alpha$.

Curves $1 \div 4$ characterize the stress distribution for the corresponding parameter 
values $\alpha$.

$$
\left(\sigma_{22}^{+}+T_{22}^{+}\right), H / \mathrm{M}^{2}
$$

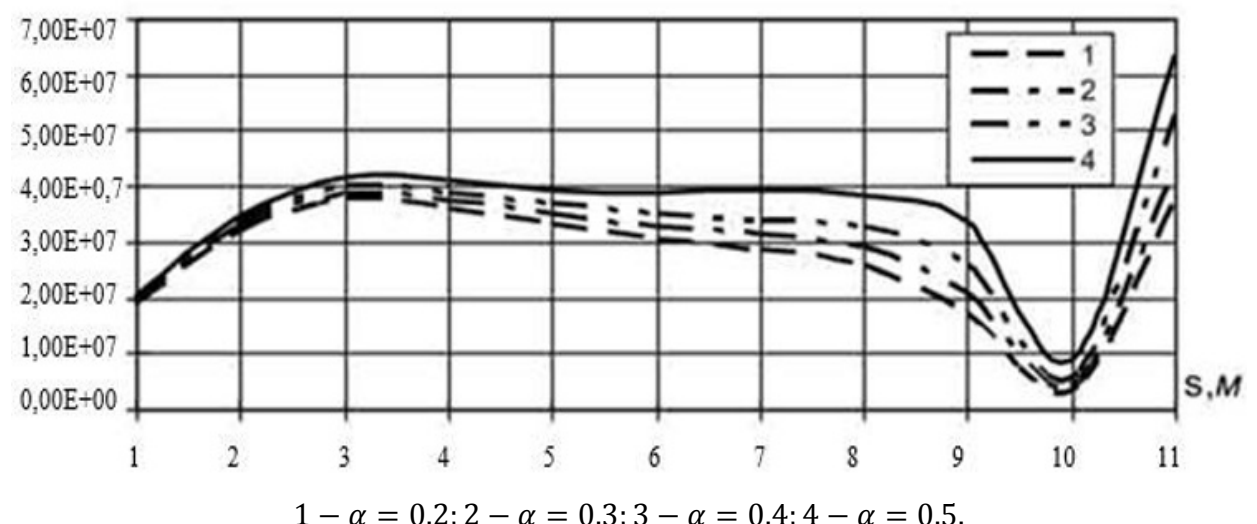

Fig. 2. Distribution $\left(\sigma_{22}^{+}+T_{22}^{+}\right)$by $s$ at the moment of time $t=5 \cdot 10^{-3} c$ at different values of the parameter $\alpha$.

The figures show the complex nature of the behavior of the shell depending on the boundary conditions under the action of mechanical and magnetic fields. It should be noted that the maximum value is observed in all cases with $s=0.5 \mathrm{M}$ and with increasing parameter $\alpha$-stress values on shell surfaces increase.

$$
\left(\sigma_{22}^{-}+T_{22}^{-}\right), H / \mathrm{M}^{2}
$$

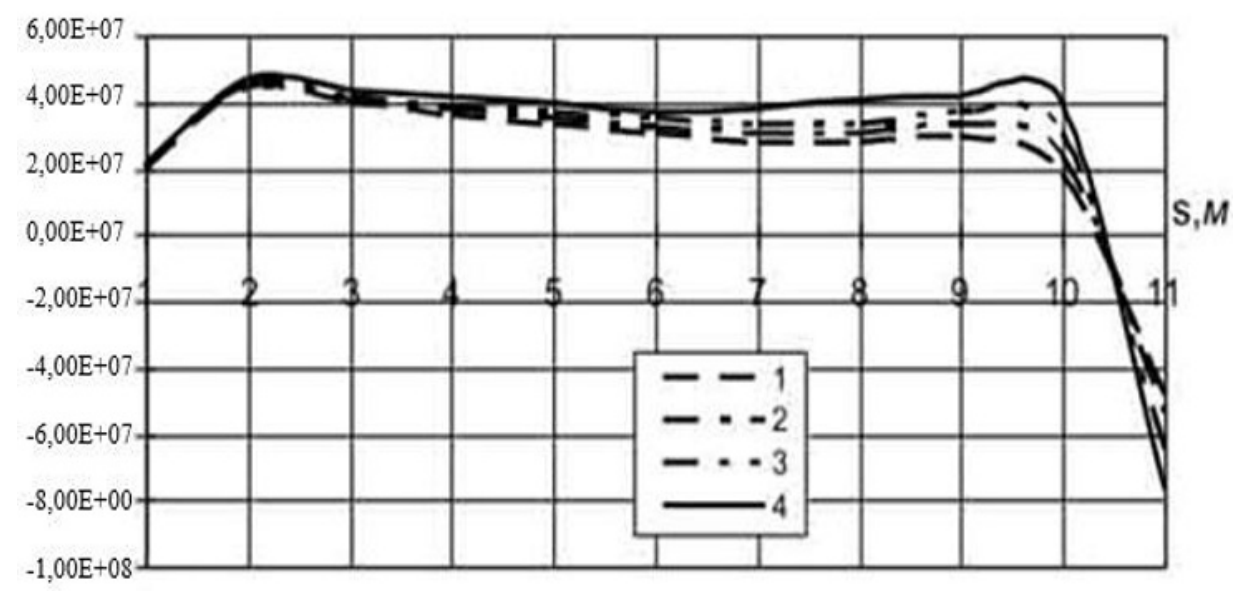

$$
1-\alpha=0.2 ; 2-\alpha=0.3 ; 3-\alpha=0.4 ; 4-\alpha=0.5 \text {. }
$$

Fig. 3. Distribution $\left(\sigma_{22}^{-}+T_{22}^{-}\right)$by $s$ at the moment of time $t=5 \cdot 10^{-3} c$ at different values of the parameter $\alpha$.

In fig. 4 and 5 are speed variations $(\partial u / \partial t)$ and accelerate $\left(\partial^{2} w / \partial t^{2}\right)$ displacement along the shell meridian at time $t=5 \cdot 10^{-3} c$ for different parameter values $\alpha$

$$
\partial u / \partial t, \mathrm{M} / \mathrm{c}
$$




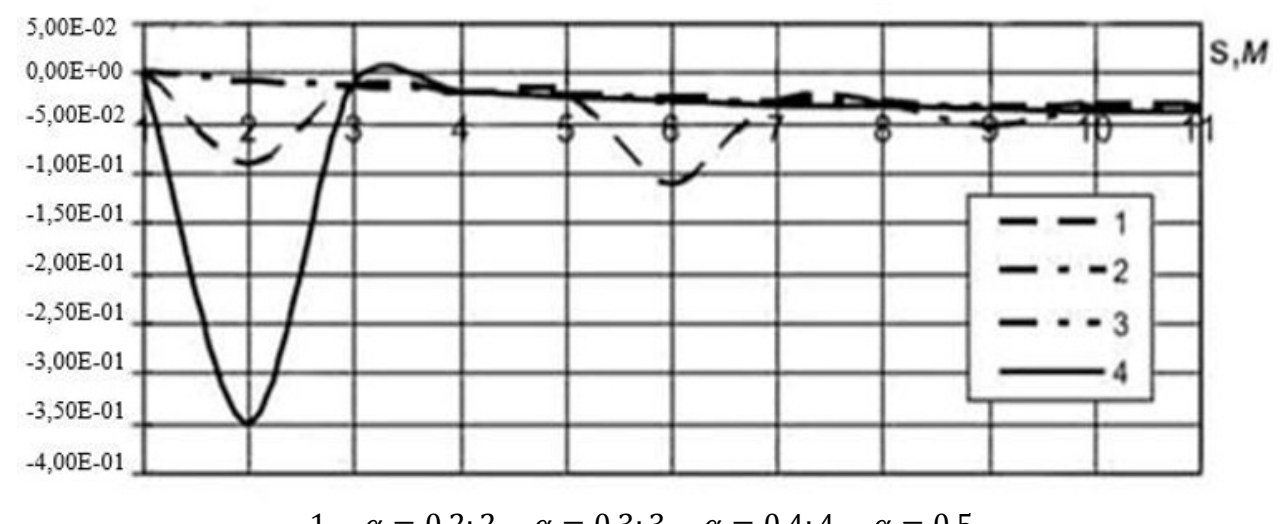

$1-\alpha=0.2 ; 2-\alpha=0.3 ; 3-\alpha=0.4 ; 4-\alpha=0.5$.

Fig. 4. Distribution $\partial u / \partial t$ by $s$ at the moment of time $t=5 \cdot 10^{-3} c$ at different values of the parameter $\alpha$.

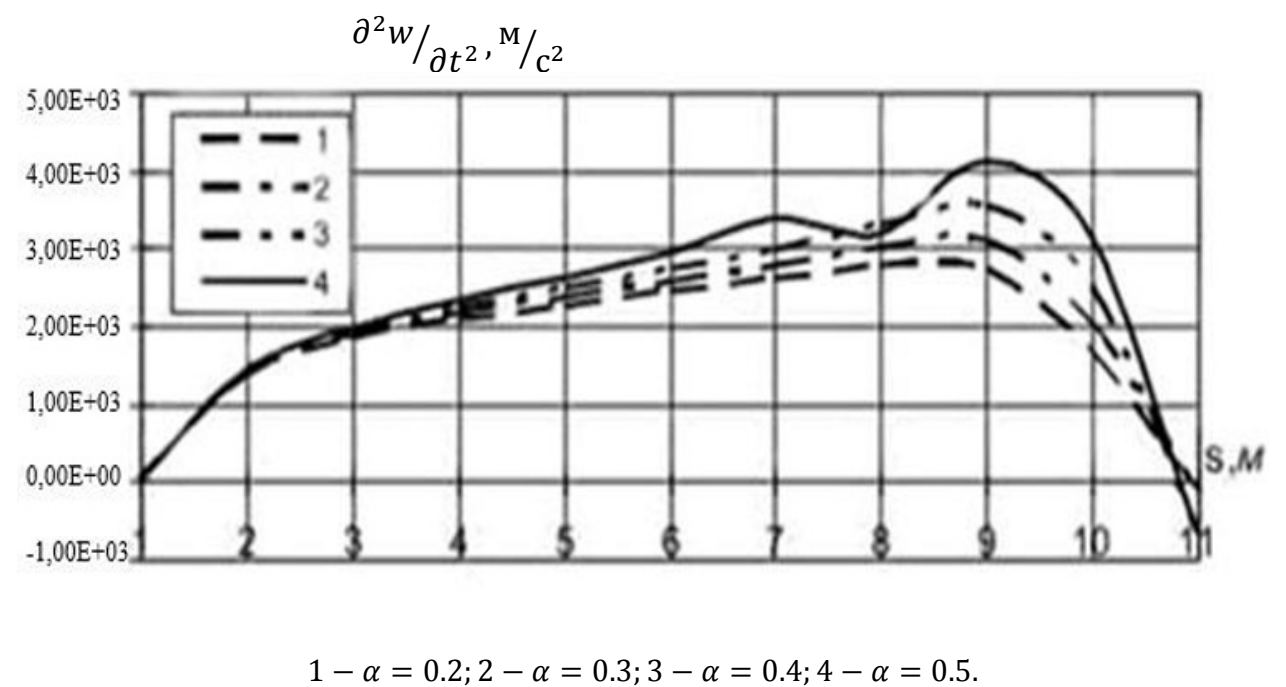

Fig. 5. Distribution $\partial^{2} w / \partial t^{2}$ by $s$ at the moment of time $t=5 \cdot 10^{-3} c$ at different values of the parameter $\alpha$.

The figures show that with increasing parameter value $\alpha$ and accordingly with a decrease in thickness $h(s)$, there is an increase in the speed of longitudinal movement along the meridian.

The maximum values of the acceleration of the radial displacement along the meridian occur when $s=0.4 \mathrm{M}$, which is associated with boundary conditions and variations in shell thickness.

\section{Conclusion}

This article discusses the related problem of magnetoelasticity for a flexible orthotropic conical shell, taking into account the orthotropic electrical conductivity. The effect of thickness on the stress-strain state of an orthotropic shell is investigated. The results obtained indicate the effect of thickness on the deformation of the shell and the need to take this factor into account in the design schemes. As can be seen, the thickness variability has 
a significant effect on changes in the stress-strain state of the shell, and taking into account the geometric nonlinearity allows us to significantly refine the pattern of deformation.

\section{References}

1. S.A. Ambartsumian, G.E. Bagdasaryan, M.V. Belubekyan. Magnetoelasticity of thin shells and plates (Science, Moscow, Russia, 1977)

2. J. Nai. Physical properties of crystals and their description using tensors and matrices( Science, Moscow, Russia, 1967)

3. Yu.I. Sirotin, M. P.Shaskolskaya. Basics of crystal physics. (Science, Moscow, Russia, 1979)

4. Y.M. Grigorenko, L.V.Molchenko. Fundamentals of the theory of plates and shells with elements of magnetoelasticity (Kiev University, 2010)

5. L.V. Mol'chenko, I.I. Loss., R.SH. Indiaminov, Int. Appl. Mech. 44, 8 (2008)

6. R.Sh. Indiaminov, International Journal Computational Technologies. 6,13 (2008)

7. L.V. Mol'chenko, I.I. Loos. Int. Appl. Mech. 46, 11(2010)

8. L.V. Mol'chenko, I.I Loos. Int. Appl. Mech. 48, 1 (2012)

9. L.V. Mol'chenko, I.I. Loos. Int. Appl. Mech. 49, 5 (2013)

10. L.V. Mol'chenko, I.I Loos. Int. Appl. Mech. 49, 3 (2013)

11. L.V. Mol'chenko, I.I. Loos, R.Sh. Indiaminov, Int. Appl. Mech. 33, 3 (1997)

12. L.V. Mol'chenko, I.I. Loos, R.Sh. Indiaminov, Int. Appl. Mech. 45, 11 (2009)

13. L.V. Mol'chenko, I.I. Loos, I.V. Plyas. Int. Appl. Mech. 46, 5 (2010)

14. L.V. Mol'chenko, I.I. Loos, I.V. Plyas, Int. Appl. Mech. 47, 3 (2011)

15. Y.M. Grigorenko, L.V. Molchenko. Fundamentals of the theory of plates and shells. (Science, Moscow, Libid, 1993).

16. Y.M. Grigorenko, L.V. Molchenko. Fundamentals of the theory of plates and shells with elements of magnetoelasticity. (Science, Moscow, Kiev University, 2010)

17. Yu.I. Sirotin, M.P. Shaskolskaya, Basics of crystal physics. (Science, 1979).

18. A.F. Ulitko, L.V. Molchenko, V.F. Kovalchuk Magnetoelasticity under dynamic loading. Training allowance. (Science, Moscow, Libsc, 1994)

19. C. Truesdeil, W. Noll. The nonlinear field theorie of mechanics (in S. Flugges Handbuch der Physic, Berlin,1960) 